\section{Access to data on humans}

SIR - The announcement that Nature intends to impose public access to data on altered animals (Nature 379, 191; 1996) is welcome. But what about unaltered humans? Can we have your help in the acquisition and deposition of linkage data on which published papers are based? The data could be accessed by e-mail after publication. This would bring human data into line with the moral leadership long provided by the nematode project.

Although the human genome has vast resources devoted to its physical mapping, there are difficulties in acquiring good data on genetic mapping. Linkage studies are published without the requirement, usual in major journals for protein and DNA sequences, of easy access to raw data. The many successful studies in mendelian disorders have their data lost to posterity after a single pair of loci have defined a linkage, often after a trawl of hundreds of markers. Studies on the non-mendelian disorders usually have insufficient information to reach the arbitrary levels of likelihood demanded without the helping hand of chance, and their massive and reliable data are not available either for the general mapping on which related advances depend or as a contribution to specific mapping in the future. Few authors seem aware of the necessary limitations of the present maps and the simple options available for their advance. As most linkage studies are analysed by a limited range of programs, compatibility is not a problem as the formats are well specified and simple.

The requirement of submission of data with manuscripts would have four advantages.

(1) It would allow data on genetic mapping to accumulate and be available for both global mapping and for scrutiny by those with special expertise in small segments of the genome.

(2) It would provide information on allelic frequencies and associations in different communities: this would give information on very close linkages as well as on the origins of populations and the mutation rates of the more mutable loci.

(3) It would allow the weak information on non-mendelian disorders, unbiased by selection for high lod scores at more than a few neighbouring loci, to be assembled together and analysed as a whole by the same programs, and also provide an incentive for program development. The vast diversion of blood and gold to linkage analysis in schizophrenia has so far led to only one hopeful segment with an allele sufficiently strong and frequent to be noticed, although in no single study is the evidence convincing.

(4) It would allow reviewers the opportunity to examine the data, and their positive recommendations would be exposed to the quality control of independent review.

In practice, the development of DNA typing in humans has been associated with a degree of secrecy new to such studies and the ethical implications have been of little interest to those preoccupied with the expanding and invasive subject of medical ethics. The sheer size of the present genetic map imposes increasing restraints on the reliability of global ordering algorithms, especially where recombinant events cannot be counted, or are not counted, while the difficulty of the subject makes it impossible for anyone to be expert on more than a few segments of the order of a thousandth of our genome.

To exploit the genome project efficiently, we need to compare the structural or physical maps, progressing well in public documentation and methods of ordering and display, with the functional or genetic maps, on which raw data are limited and methods of ordering and display less advanced.

\section{J. H. Edwards}

Department of Genetics, University of Oxford,

Department of Genetics, Oxford OX1 3QU, UK
If you've ever developed a purification strategy by yourself, you know there is a lot to consider. Creating media screening schemes; designing buffer preparation routines; selecting which columns to use- - even listing the tasks takes careful consideration. But now there's a better way of working. screening. You'll save time as the system automatically recommends the best columns for your runs. You get fast $\mathrm{pH}$ screening as it automatically prepares your buffers from stock solutions. The moment you turn ÄKTAexplorer on, you're presented with a direct path to full-scale purification.

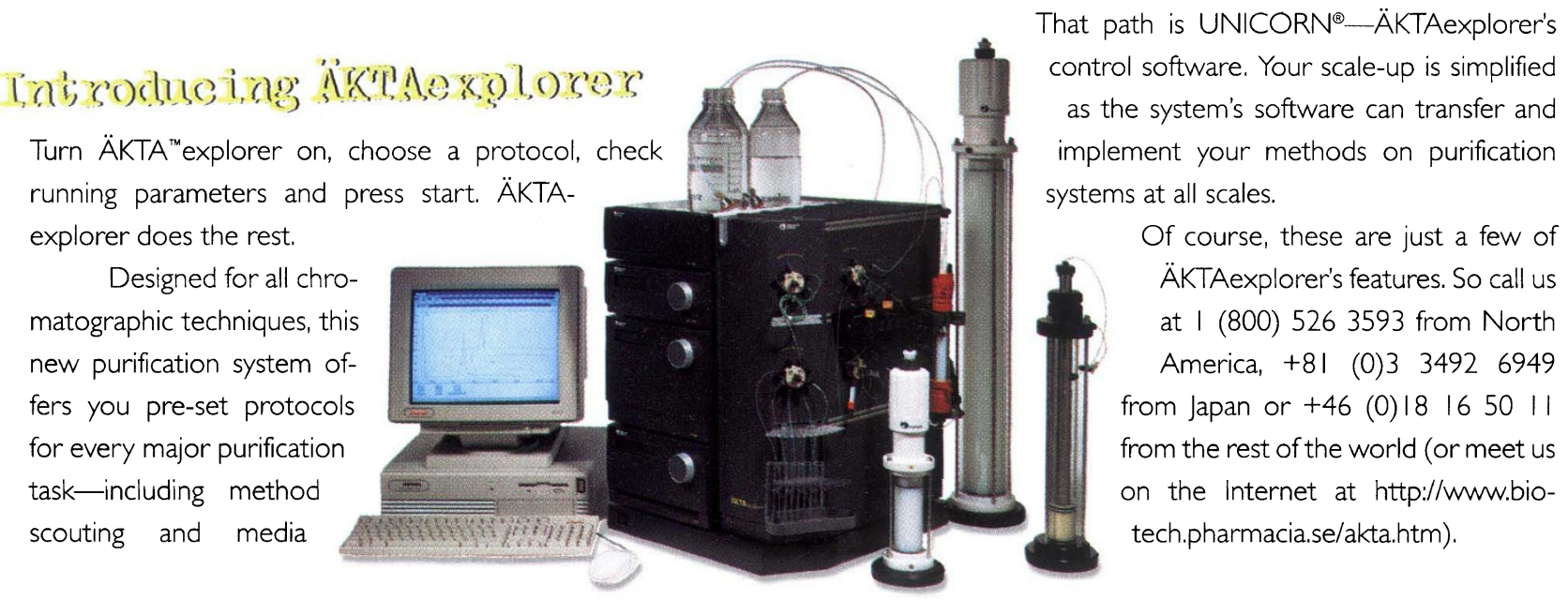

